\title{
ON THE MAXIMUM DENSITY OF MINIMAL ASYMPTOTIC BASES
}

\author{
MELVYN B. NATHANSON AND ANDRÁS SÁRKÖZY
}

(Communicated by Larry J. Goldstein)

\begin{abstract}
A set $A$ of nonnegative integers is an asymptotic basis of order $h$ if every sufficiently large integer is the sum of $h$ elements of $A$. It is proved that if $A$ is an asymptotic basis of order $h$ with lower asymptotic density $d_{L}(A)>1 / h$, then there is a set $W$ contained in $A$ such that $W$ has positive asymptotic density and $A \backslash W$ is an asymptotic basis of order $h$. This implies that if $A$ is a minimal asymptotic basis of order $h$, then $d_{L}(A) \leq 1 / h$.
\end{abstract}

Let $A$ be a subset of the nonnegative integers $N$. Denote by $h A$ the set of all numbers $n$ that can be written in the form $n=a_{1}+\cdots+a_{h}$, where $a_{i} \in A$ for $i=1, \ldots, h$ and the $a_{i}$ are not necessarily distinct. The set $A$ is a basis of order $h$ if $h A=N$. If $h A$ contains every sufficiently large integer, then $A$ is an asymptotic basis of order $h$. Much of classical additive number theory is the study of special sets $A$ that are bases or asymptotic bases of order $h$ for some $h \geq 2$.

Minimal asymptotic bases form an important extremal class in additive number theory. The asymptotic basis $A$ of order $h$ is minimal if no proper subset of $A$ is an asymptotic basis of order $h$. It follows that if $A$ is minimal, then for every element $a \in A$ there must be infinitely many positive integers $n$, each of whose representations as a sum of $h$ elements of $A$ includes the number $a$ as a summand. Stöhr [7] introduced the concept of minimal asymptotic basis, and Härtter [3] proved that minimal asymptotic bases of order $h$ exist for all $h \geq 2$. Erdös and Nathanson [1] survey recent results on minimal asymptotic bases.

For any set $A$ of integers, the counting function of $A$, denoted $A(x)$, is defined by $A(x)=\operatorname{card}(\{a \in A \mid 1 \leq a \leq x\})$. The lower asymptotic density of $A$, denoted $d_{L}(A)$, is defined by $d_{L}(A)=\liminf A(x) / x$. If $\alpha=\lim A(x) / x$ exists, then $\alpha$ is called the asymptotic density of $A$, and denoted $d(A)$.

For every $h \geq 2$, Nathanson [5, 6] has constructed minimal asymptotic bases $A$ of order $h$ that satisfy $A(x) \ll x^{1 / h}$. Since $A(x) \gg x^{1 / h}$ for every

Received by the editors December 12, 1986 and, in revised form, January 11, 1988.

1980 Mathematics Subject Classification (1985 Revision). Primary 11B13, 11 B05, 11 P99.

Key words and phrases. Minimal asymptotic bases, additive bases, sumsets, additive number theory. 
asymptotic basis $A$ of order $h$, it follows that these examples are the "thinnest" possible minimal asymptotic bases. Erdös and Nathanson [2] have recently constructed minimal asymptotic bases $A$ of order $h$ with $d(A)=1 / h$. In this paper we prove that these are the "fattest" possible minimal asymptotic bases, in the sense that $d_{L}(A) \leq 1 / h$ for every minimal asymptotic basis $A$ of order $h$. The proof uses a deep result of Kneser on the lower asymptotic density of sums of sets of integers.

Let $A$ and $B$ be sets of nonnegative integers. We shall write $A \sim B$ if $A$ and $B$ coincide for all sufficiently large integers. For $g \geq 1$, let $B^{(g)}$ be the set of all integers $t$ such that $t \equiv b(\bmod g)$ for some $b \in B$. Let $h \geq 2$. Kneser [4] proved that either $d_{L}(h B) \geq h d_{L}(B)$ or there exists an integer $g \geq 1$ such that $h B \sim h B^{(g)}$ and $d_{L}(h B) \geq h d_{L}(B)-(h-1) / g$.

Theorem 1. Let $h \geq 2$ and let $A$ be an asymptotic basis of order $h$. If $B \subseteq A$ and $d_{L}(B)>1 / h$, then there is a finite set $F \subseteq A \backslash B$ such that $B \cup F$ is an asymptotic basis of order $h$.

Proof. Assume that $B$ is not an asymptotic basis of order $h$. Then $h B \nsim N$. Since $d_{L}(h B) \leq 1<h d_{L}(B)$, Kneser's theorem implies that there exists an integer $g \geq 1$ such that $h B^{(g)} \sim h B \nsim N$ and

$$
h d_{L}(B)-(h-1) / g \leq d_{L}(h B)=d\left(h B^{(g)}\right) .
$$

Since $h B^{(g)}$ is a union of congruence classes modulo $g$, it follows from $h B^{(g)} \nsim$ $N$ that $d\left(h B^{(g)}\right)=r / g \leq 1-1 / g$. Let $d_{L}(B)=1 / h+\delta$. Then $\delta>0$, and $1+h \delta-(h-1) / g \leq 1-1 / g$, hence

$$
1 \leq g \leq(h-2) /(h \delta) \text {. }
$$

Note that $B \subseteq B^{(g)}$ and $B \subseteq A$. If $A \subseteq B^{(g)}$, then $h B \subseteq h A \subseteq h B^{(g)}$. Since $A$ is an asymptotic basis of order $h$, it follows that $N \sim h A \sim h B^{(g)} \sim h B$, which contradicts the hypothesis that $B$ is not an asymptotic basis of order $h$. Therefore, $A \nsubseteq B^{(g)}$ and there exists an integer $c_{1} \in A$ such that $c_{1} \not \equiv b$ $(\bmod g)$ for every $b \in B$. Let $g_{1}=g$.

Let $B_{1}=B \cup\left\{c_{1}\right\}$. If $B_{1}$ is an asymptotic basis of order $h$, let $F=\left\{c_{1}\right\}$. If $B_{1}$ is not an asymptotic basis of order $h$, there exists an integer $g_{2}$ satisfying (*) and an integer $c_{2} \in A$ such that $c_{2} \not \equiv b\left(\bmod g_{2}\right)$ for all $b \in B_{1}$. Let $B_{2}=B_{1} \cup\left\{c_{2}\right\}=B \cup\left\{c_{1}, c_{2}\right\}$.

Continuing inductively, we obtain a sequence of integers $g_{1}, g_{2}, \ldots$ satisfying (*) and a sequence $c_{1}, c_{2}, \ldots$ of elements of $A$ such that $c_{i} \not \equiv b\left(\bmod g_{i}\right)$ for all $b \in B_{i-1}=B \cup\left\{c_{1}, c_{2}, \ldots, c_{i-1}\right\}$. Since there are only finitely many distinct congruence classes with respect to moduli bounded above by $(h-2) /(h \delta)$, the inductive process must terminate in a finite number of steps, and we obtain a finite set $F=\left\{c_{1}, c_{2}, \ldots\right\} \subseteq A$ such that $B \cup F$ is an asymptotic basis of order $h$. This completes the proof.

Theorem 2. Let $h \geq 2$, and let $A$ be an asymptotic basis of order $h$ with $d_{L}(A)=1 / h+\delta$, where $\delta>0$. Let $0<\tau<\delta$. Then there is a set $W \subseteq A$ with 
asymptotic density $d(W)=\tau$ such that $A \backslash W$ is an asymptotic basis of order $h$.

Proof. Let $W^{\prime}$ be any subset of $A$ with $d\left(W^{\prime}\right)=\tau$. Let $B=A \backslash W^{\prime}$. Then $d_{L}(B)=1 / h+\delta-\tau>1 / h$. By Theorem 1 , there is a finite set $F \subseteq A \backslash B=W^{\prime}$ such that $B \cup F=A \backslash\left(W^{\prime} \backslash F\right)$ is an asymptotic basis of order $h$. Let $W=$ $W^{\prime} \backslash F$. Then $d(W)=d\left(W^{\prime}\right)=\tau$. This proves the theorem.

Theorem 3. Let $A$ be a minimal asymptotic basis of order $h$. Then $d_{L}(A) \leq$ $1 / h$.

Proof. If $d_{L}(A)>1 / h$, then by Theorem 2 there is a set $W \subseteq A$ with $d(W)>$ 0 such that $A \backslash W$ is an asymptotic basis of order $h$, and this contradicts the minimality of $A$.

\section{REFERENCES}

1. P. Erdös and M. B. Nathanson, Problems and results on minimal bases in additive number theory, in: Number Theory, New York 1985-86, Lecture Notes in Math., vol. 1240, SpringerVerlag, Heidelberg, 1987, pp. 87-96.

2. _ , and _ Minimal asymptotic bases with prescribed densities, Illinois J. Math. 32 (1988), to appear.

3. E. Härtter, Ein Beitrag zur Theorie der Minimalbasen, J. Reine Angew. Math. 196 (1956), 170-204.

4. M. Kneser, Abschätzung der asymptotischen Dichte von Summenmengen, Math. Z. 58 (1953), 459-484.

5. M. B. Nathanson, Minimal bases and maximal nonbases in additive number theory, J. Number Theory 6 (1974), 324-333.

6. __. Minimal bases and powers of 2, Acta Arith. 51 (1988), 95-102.

7. A. Stöhr, Gelöste und ungelöste Fragen über Basen der natürlichen Zahlenreihe. II, J. Reine Angew. Math. 194 (1955), 111-140.

Provost and Vice President for Academic Affairs, lehman College (CUNY), Bronx, NEW YORK 10468

Department of Mathematics, Baruch College (CUNY), New York, New York 10010 Current address of A. Sárköty:

Mathematical Institute of the Hungarian Academy of Sciences, Budapest, Hungary 